\title{
Bivariate Empirical Mode Decomposition for ECG-based Biometric Identification with Emotional Data*
}

\author{
Hany Ferdinando, Tapio Seppänen, Esko Alasaarela
}

\begin{abstract}
Emotions modulate ECG signals such that they might affect ECG-based biometric identification in real life application. It motivated in finding good feature extraction methods where the emotional state of the subjects has minimum impacts. This paper evaluates feature extraction based on bivariate empirical mode decomposition (BEMD) for biometric identification when emotion is considered. Using the ECG signal from the Mahnob-HCI database for affect recognition, the features were statistical distributions of dominant frequency after applying BEMD analysis to ECG signals. The achieved accuracy was $99.5 \%$ with high consistency using $\mathrm{kNN}$ classifier in 10 -fold cross validation to identify 26 subjects when the emotional states of the subjects were ignored. When the emotional states of the subject were considered, the proposed method also delivered high accuracy, around $99.4 \%$. We concluded that the proposed method offers emotion-independent features for ECG-based biometric identification. The proposed method needs more evaluation related to testing with other classifier and variation in ECG signals, e.g. normal ECG vs. ECG with arrhythmias, ECG from various ages, and ECG from other affective databases.
\end{abstract}

\section{INTRODUCTION}

Previous studies have reported many attempts to use ECG signal in biometric identification using various method with good results because ECG waveforms have unique identity properties for human identification [1]. Biometric identification using ECG has at least two advantages, i.e. it is suitable for disabled people [2] and it is non-invasive. One of the challenges in ECG-based biometric identification is lack of standard for fiducial points-based feature extraction because of its uniqueness among the subject and various emotional and physiological condition [3] [4]. This paper focuses on the evaluation of the proposed feature extraction method under different emotional state of the subjects for ECG-based biometric identification.

Lugovaya [5] studied the ECG biometric identification from 90 subjects where the ECGs were recorded in the sitting position and the emotional states were ignored. Although it achieved $96 \%$ identification rate, there was no analysis related to the emotional state of the subjects.

*Research supported by the Directorate General of Higher Education, Ministry of Research and Higher Education the Republic of Indonesia and the Finnish Cultural Foundation the North Ostrobothnia Regional Fund.

Hany Ferdinando is with the Optoelectronics and Measurement Technique unit, University of Oulu, Finland and Department of Electrical Engineering, Petra Christian University, Indonesia. (phone: +358 294 482765, fax: +358 8553 2774, e-mail: hferdina@ee.oulu.fi).

Tapio Seppånen is with the Physiological signal analysis team, University of Oulu, Finland (e-mail: tapio@ee.oulu.fi).

Esko Alasaarela is with the Optoelectronics and Measurement Technique unit, University of Oulu, Finland (e-mail: esko.alasaarela@ee.oulu.fi).
Page et al. [6] utilizing deep neural nets to authenticate 90 subjects by analyzing single QRS complex. It achieved accuracy around $99.5 \%$. This study used ECG measured during resting state, which was not practical in real situation as the emotional state of the people is dynamic, although it required single heart beat only for identification.

To our knowledge, previous studies have not dealt with emotional state of the subjects. This issue is important because emotions change dynamically in daily life and they also effect the fiducial points of the ECG. Agrafioti [3] showed how the perils of ignoring time dependency on the ECG signal. A new feature extraction method is needed to compensate the effect of emotions to ECG signals.

This study aims to contribute to this growing research area by exploring the feature extraction method based on the bivariate empirical mode decomposition (BEMD) analysis for ECG-based biometric identification and evaluate it under different emotional conditions. For this reason, we used ECG signals from the Mahnob-HCI database for affective recognition [7] to complement the ECG-based biometric identification study.

\section{MATERIALS AND METHODS}

\section{A. The Database}

The Mahnob-HCI database for affective recognition involved 27 healthy subjects in the synchronized measurement of EEG, several peripheral physiological signals (ECG, respiration, temperature, skin conductance), eye gaze, body and face videos while being emotionally stimulated by pictures and videos [7]. This database was chosen because the signals can be considered as signals in real life situation where the emotional state of people changes dynamically. However, this study ignored abnormal ECG signal.

The ECG signals in the Mahnob-HCI database was downloaded under the group name of "Selection of Emotion Elicitation" with 513 samples from 26 subject because ECGs from one subject were corrupted. Later, sample from session 2508 was discarded as well with the same reason. Each ECG signal consists of emotionally stimulated and unstimulated signal. The experiments used ECG signals from the stimulated phase only with signal pre-processing to remove motion artifact and power line interference [7].

\section{B. Feature Extraction}

Fig. 1 shows the block diagram of the system. It starts with analyzing the signal using BEMD [8] which requires a complex value signal. The original signal serves as real part while the synthetic ECG signal, generated by a model developed by McSharry et al. [9] synchronized based on the R-wave event detected using Pan-Tompkins method [10], as 
imaginary part. As suggested in [11], it is recommended to use a one-cycle synthetic ECG signal as a template and put it based on the R-wave event because it is faster than generating one-cycle ECG for each detected R-wave event.

Since the BEMD is sensitive to signal length and ECG signals from the database have different length, each ECG signal was divided into 5-second segments because preliminary experiments show that a 5 seconds ECG signal results 5-6 intrinsic mode functions (IMFs) plus residual [11] [12]. We are interested to get several dominant frequencies (DFs), estimated using spectrogram analysis with window size of $30,50,100,150,200,250$ and 300 samples and overlap parameters of $10 \%$ to $90 \%$ (with $10 \%$ steps) [11], from the first three IMFs because they tend to preserve information from the QRS complex [13].

The proposed new features are based on the statistical distribution (mean, standard deviation, median, Q1, Q3, IQR, skewness, kurtosis, percentile 2.5 , percentile 10 , percentile 90, percentile 97.5, maximum, and minimum) of DFs and their first difference, resulting 168 features. The most discriminant features are selected by applying a sequential forward-floating search process. The final number of selected features varies from 15 to 109 depending on the window size and overlap parameters in the spectrogram analysis. We also applied supervised dimensionality reduction with NCA as in [14] to limit all feature vectors dimensions to 15 , and then compared classification accuracy with the ones without dimensionality reduction.

\section{Classifier and Validation Method}

The samples were divided into training, testing and validation part: $20 \%$ of the samples were held out for validation, while the rest of them were subject to training and testing with 10 -fold cross validation using $\mathrm{kNN}$ for person identification. There were 100 iterations for each $\mathrm{k}$, the number of nearest neighbors, with a random sampling of data for training, testing and validation set. The reported accuracies are the average over the repetition.

During the person identification test runs, the discrete emotional labels (neutral, anger, disgust, fear, joy, sadness, surprise, amusement, and anxiety) and the 3-level sublabels of valence and arousal for each test sample were recorded to calculate the identification accuracy for each emotional label. The accuracy for an emotional label was defined as the ratio of true person identifications to all identifications using data only from that emotional label. Means and variances are calculated for the accuracy with low variance indicating high consistency in the emotional class.

\section{RESULTS AND DISCUSSIONS}

Table I shows the grid search of window size and overlap parameters used in spectrogram analysis. The proposed feature extraction method offers good results as the accuracies were higher than $90 \%$ with chance level for 26 subjects was around $3.8 \%$. The shaded cells represent the parameter combinations having good accuracies. Having single digit of variance, the proposed method also demonstrates high consistency.

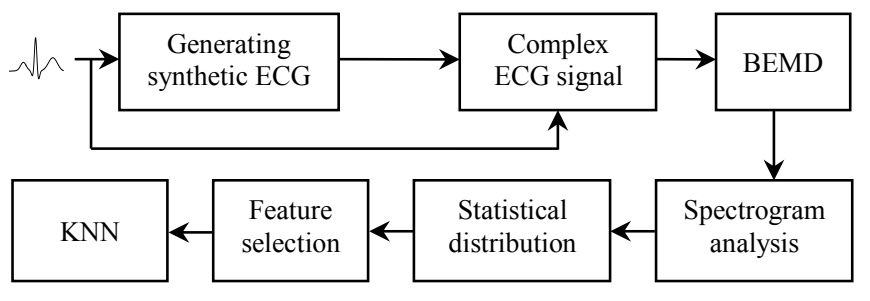

Figure 1. Block diagram of the system [11]

Since the values in the shaded cells were close to each other, the Law of Large Numbers (LLN) was applied to estimate the true accuracy with 1000 iterations. Table II shows the best five results after the LLN.

A significant test using t-test with alpha 0.05 was conducted to evaluate if difference was significant with either $99.5 \pm 0.7$ (250 samples and $90 \%$ overlap) or $99.5 \pm 0.8$ (200 samples and $90 \%$ overlap) as a reference. For the two references had no significant difference, the 250 samples and $90 \%$ overlap was chosen because of its computational speed. The p-values for rest of the results were very close to zero, indicating that the difference to the reference value was significant and the best performance was $99.5 \pm 0.7$. The best five results above occurred at the large overlap, indicating that very fine spectrogram analysis was needed while the window size parameter had no significant impact.

The performance of the proposed feature extraction method was also evaluated based on the emotional state of the subject in valence and arousal with 1000 repetitions for

TABLE I. ACCURACY OF THE SYSTEM PRESENTED AS GRID SEARCH RESULTS

\begin{tabular}{|c|c|c|c|c|c|c|c|c|}
\hline & \multicolumn{7}{|c|}{ Window size (samples) } \\
\hline & & 30 & 50 & 100 & 150 & 200 & 250 & 300 \\
\hline \multirow{9}{*}{ 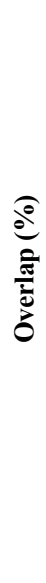 } & 10 & $\begin{array}{l}93.5 \\
\pm 2.2\end{array}$ & $\begin{array}{l}95.8 \\
\pm 2.0\end{array}$ & $\begin{array}{l}95.7 \\
\pm 1.9\end{array}$ & $\begin{array}{l}97.3 \\
\pm 1.7\end{array}$ & $\begin{array}{l}97.0 \\
\pm 1.8\end{array}$ & $\begin{array}{l}93.1 \\
\pm 2.1\end{array}$ & $\begin{array}{l}94.9 \\
\pm 2.1\end{array}$ \\
\hline & 20 & $\begin{array}{l}95.6 \\
\pm 2.1 \\
\end{array}$ & $\begin{array}{l}97.4 \\
\pm 1.6\end{array}$ & $\begin{array}{l}96.7 \\
\pm 1.6\end{array}$ & $\begin{array}{l}97.8 \\
\pm 1.3\end{array}$ & $\begin{array}{l}97.6 \\
\pm 1.2 \\
\end{array}$ & $\begin{array}{l}95.0 \\
\pm 2.2\end{array}$ & $\begin{array}{l}96.9 \\
\pm 1.8 \\
\end{array}$ \\
\hline & 30 & $\begin{array}{l}96.5 \\
\pm 2.1\end{array}$ & $\begin{array}{l}96.2 \\
\pm 1.7\end{array}$ & $\begin{array}{l}97.7 \\
\pm 1.5\end{array}$ & $\begin{array}{l}97.1 \\
\pm 1.6\end{array}$ & $\begin{array}{l}97.8 \\
\pm 1.6\end{array}$ & $\begin{array}{l}97.8 \\
\pm 1.5\end{array}$ & $\begin{array}{l}96.3 \\
\pm 1.7\end{array}$ \\
\hline & 40 & $\begin{array}{l}96.4 \\
\pm 1.9 \\
\end{array}$ & $\begin{array}{l}96.6 \\
\pm 1.4 \\
\end{array}$ & $\begin{array}{l}98.6 \\
\pm 1.3 \\
\end{array}$ & $\begin{array}{l}98.2 \\
\pm 1.3 \\
\end{array}$ & $\begin{array}{l}98.9 \\
\pm 1.1 \\
\end{array}$ & $\begin{array}{l}98.6 \\
\pm 1.0 \\
\end{array}$ & $\begin{array}{l}97.8 \\
\pm 1.5 \\
\end{array}$ \\
\hline & 50 & $\begin{array}{l}96.7 \\
\pm 1.7\end{array}$ & $\begin{array}{l}97.9 \\
\pm 1.5\end{array}$ & $\begin{array}{l}99.0 \\
\pm 1.1\end{array}$ & $\begin{array}{l}98.0 \\
\pm 1.4\end{array}$ & $\begin{array}{l}98.5 \\
\pm 1.3\end{array}$ & $\begin{array}{l}99.0 \\
\pm 1.0\end{array}$ & $\begin{array}{l}98.0 \\
\pm 1.3 \\
\end{array}$ \\
\hline & 60 & $\begin{array}{l}98.2 \\
\pm 1.2\end{array}$ & $\begin{array}{l}99.0 \\
\pm 1.1 \\
\end{array}$ & $\begin{array}{l}99.1 \\
\pm 1.0\end{array}$ & $\begin{array}{l}98.6 \\
\pm 1.3\end{array}$ & $\begin{array}{l}98.8 \\
\pm 1.0\end{array}$ & $\begin{array}{l}99.3 \\
\pm 0.9\end{array}$ & $\begin{array}{l}98.6 \\
\pm 1.0 \\
\end{array}$ \\
\hline & 70 & $\begin{array}{l}98.5 \\
\pm 1.2\end{array}$ & $\begin{array}{l}98.9 \\
\pm 1.2\end{array}$ & $\begin{array}{l}99.2 \\
\pm 0.8\end{array}$ & $\begin{array}{l}98.6 \\
\pm 1.1\end{array}$ & $\begin{array}{l}99.4 \\
\pm 0.8\end{array}$ & $\begin{array}{l}99.3 \\
\pm 0.8\end{array}$ & $\begin{array}{l}98.7 \\
\pm 1.1\end{array}$ \\
\hline & 80 & $\begin{array}{l}99.0 \\
\pm 1.1\end{array}$ & $\begin{array}{l}98.9 \\
\pm 1.1\end{array}$ & $\begin{array}{l}98.9 \\
\pm 1.2\end{array}$ & $\begin{array}{l}99.2 \\
\pm 0.9\end{array}$ & $\begin{array}{l}99.4 \\
\pm 0.8\end{array}$ & $\begin{array}{l}99.3 \\
\pm 0.8\end{array}$ & $\begin{array}{l}99.0 \\
\pm 0.9 \\
\end{array}$ \\
\hline & 90 & $\begin{array}{l}98.9 \\
\pm 0.9\end{array}$ & $\begin{array}{l}98.9 \\
\pm 1.2\end{array}$ & $\begin{array}{l}99.3 \\
\pm 0.9\end{array}$ & $\begin{array}{l}99.3 \\
\pm 0.8\end{array}$ & $\begin{array}{l}99.6 \\
\pm 0.6\end{array}$ & $\begin{array}{l}99.4 \\
\pm 0.9\end{array}$ & $\begin{array}{l}99.2 \\
\pm 0.9\end{array}$ \\
\hline
\end{tabular}

TABLE II. ESTIMATED TRUE ACCURACY USING THE LAW OF LARGE NUMBERS

\begin{tabular}{|c|c|c|}
\hline \multicolumn{2}{|c|}{ Spectrogram Parameters } & \multirow{2}{*}{$\begin{array}{c}\text { Accuracy } \\
(\%)\end{array}$} \\
\hline $\begin{array}{l}\text { Window Size } \\
\text { (samples) }\end{array}$ & $\begin{array}{c}\text { Overlap } \\
\text { (\%) }\end{array}$ & \\
\hline 100 & 90 & $99.3 \pm 0.9$ \\
\hline 200 & 90 & $99.5 \pm 0.8$ \\
\hline 250 & 80 & $99.3 \pm 0.9$ \\
\hline 250 & 90 & $99.5 \pm 0.7$ \\
\hline 300 & 90 & $99.3 \pm 0.8$ \\
\hline
\end{tabular}


each parameter combination. Table III and IV display the average accuracy over 3-level sub-labels of valence and arousal respectively. The average accuracies of the whole experiments were $97.9 \pm 1.4$ for valence and $97.8 \pm 1.4$ for arousal.

Shaded cells in Table III and IV mark parameter combination with the best result. Most of the results have small variance indicating that the performance of each sublabel within valence and arousal were close to each other. These experiments confirmed that the proposed feature extraction method is independent to valence and arousal state of the subject.

Fig. 2 and 3 compare the accuracy among the parameter combinations. The worst performance for overlap parameter on each window size in both valence and arousal occurred at $10 \%$ while the higher overlap offered better performance. Generally, $90 \%$ overlap outperformed the other by giving performance higher than $98 \%$ for both valence and arousal.

TABLE III. AVERAGE PERFORMANCES WHEN VALENCE STATES OF THE SUBJECT WERE CONSIDERED

\begin{tabular}{|c|c|c|c|c|c|c|c|c|}
\hline & \multicolumn{7}{|c|}{ Window size (samples) } \\
\hline & & 30 & 50 & 100 & 150 & 200 & 250 & 300 \\
\hline \multirow{9}{*}{ 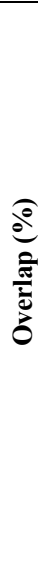 } & 10 & $\begin{array}{r}94.0 \\
\pm 2.4\end{array}$ & $\begin{array}{r}95.9 \\
\pm 1.1\end{array}$ & $\begin{array}{r}95.6 \\
\pm 0.7\end{array}$ & $\begin{array}{r}96.9 \\
\pm 0.3\end{array}$ & $\begin{array}{r}96.8 \\
\pm 0.2\end{array}$ & $\begin{array}{c}93.4 \\
\pm 0.4\end{array}$ & $\begin{array}{r}95.5 \\
\pm 2.1\end{array}$ \\
\hline & 20 & $\begin{array}{r}95.8 \\
\pm 2.0\end{array}$ & $\begin{array}{c}97.4 \\
\pm 0.6\end{array}$ & $\begin{array}{r}96.9 \\
\pm 1.0\end{array}$ & $\begin{array}{c}98.1 \\
\pm 0.8\end{array}$ & $\begin{array}{r}97.6 \\
\pm 1.1\end{array}$ & $\begin{array}{r}95.4 \\
\pm 1.7\end{array}$ & $\begin{array}{r}96.9 \\
\pm 1.8\end{array}$ \\
\hline & 30 & $\begin{array}{r}96.9 \\
\pm 1.3\end{array}$ & $\begin{array}{r}95.9 \\
\pm 0.8\end{array}$ & $\begin{array}{c}97.8 \\
\pm 0.8\end{array}$ & $\begin{array}{c}97.1 \\
\pm 1.8\end{array}$ & $\begin{array}{r}97.9 \\
\pm 0.4\end{array}$ & $\begin{array}{c}97.8 \\
\pm 0.7\end{array}$ & $\begin{array}{r}95.9 \\
\pm 0.3\end{array}$ \\
\hline & 40 & $\begin{array}{r}96.9 \\
\pm 1.4\end{array}$ & $\begin{array}{r}96.5 \\
\pm 0.4\end{array}$ & $\begin{array}{r}98.5 \\
\pm 0.6\end{array}$ & $\begin{array}{r}98.0 \\
\pm 0.8\end{array}$ & $\begin{array}{c}98.7 \\
\pm 0.4\end{array}$ & $\begin{array}{r}98.4 \\
\pm 0.5\end{array}$ & $\begin{array}{r}97.6 \\
\pm 1.2\end{array}$ \\
\hline & 50 & $\begin{array}{r}96.9 \\
\pm 1.3\end{array}$ & $\begin{array}{r}97.9 \\
\pm 0.8\end{array}$ & $\begin{array}{r}98.8 \\
\pm 0.3\end{array}$ & $\begin{array}{r}97.8 \\
\pm 1.2\end{array}$ & $\begin{array}{r}98.6 \\
\pm 0.7\end{array}$ & $\begin{array}{r}98.6 \\
\pm 0.2\end{array}$ & $\begin{array}{c}97.7 \\
\pm 0.6\end{array}$ \\
\hline & 60 & $\begin{array}{r}98.2 \\
\pm 0.4\end{array}$ & $\begin{array}{c}99.1 \\
\pm 0.4\end{array}$ & $\begin{array}{r}99.0 \\
\pm 0.4\end{array}$ & $\begin{array}{r}98.6 \\
\pm 0.9\end{array}$ & $\begin{array}{r}98.6 \\
\pm 0.6 \\
\end{array}$ & $\begin{array}{r}98.7 \\
\pm 0.3 \\
\end{array}$ & $\begin{array}{r}98.6 \\
\pm 0.1 \\
\end{array}$ \\
\hline & 70 & $\begin{array}{r}98.6 \\
\pm 0.8\end{array}$ & $\begin{array}{r}98.8 \\
\pm 0.5\end{array}$ & $\begin{array}{r}99.2 \\
\pm 0.3\end{array}$ & $\begin{array}{r}98.6 \\
\pm 0.1\end{array}$ & $\begin{array}{r}99.2 \\
\pm 0.3\end{array}$ & $\begin{array}{c}99.3 \\
\pm 0.1\end{array}$ & $\begin{array}{c}98.8 \\
\pm 0.1\end{array}$ \\
\hline & 80 & $\begin{array}{r}98.9 \\
\pm 0.4\end{array}$ & $\begin{array}{r}99.1 \\
\pm 0.5\end{array}$ & $\begin{array}{r}98.9 \\
\pm 0.3\end{array}$ & $\begin{array}{r}99.1 \\
\pm 0.5\end{array}$ & $\begin{array}{c}99.1 \\
\pm 0.1\end{array}$ & $\begin{array}{c}99.3 \\
\pm 0.1\end{array}$ & $\begin{array}{r}98.9 \\
\pm 0.3\end{array}$ \\
\hline & 90 & $\begin{array}{c}99.1 \\
\pm 0.5\end{array}$ & $\begin{array}{r}98.9 \\
\pm 0.2\end{array}$ & $\begin{array}{c}99.3 \\
\pm 0.4\end{array}$ & $\begin{array}{c}99.2 \\
\pm 0.2\end{array}$ & $\begin{array}{c}99.4 \\
\pm 0.1\end{array}$ & $\begin{array}{c}99.4 \\
\pm 0.1\end{array}$ & $\begin{array}{r}99.4 \\
\pm 0.3\end{array}$ \\
\hline
\end{tabular}

TABLE IV. AVERAGE PERFORMANCES WHEN AROUSAL STATES OF THE SUBJECT WERE CONSIDERED

\begin{tabular}{|c|c|c|c|c|c|c|c|c|}
\hline & \multicolumn{7}{|c|}{ Window size (samples) } \\
\hline & & 30 & 50 & 100 & 150 & 200 & 250 & 300 \\
\hline \multirow{9}{*}{$\frac{\hat{0}}{\frac{\hat{\sigma}}{2}}$} & 10 & $\begin{array}{r}93.7 \\
\pm 2.0\end{array}$ & $\begin{array}{r}95.5 \\
\pm 2.1\end{array}$ & $\begin{array}{r}95.2 \\
\pm 1.6\end{array}$ & $\begin{array}{r}97.0 \\
\pm 0.5\end{array}$ & $\begin{array}{r}96.8 \\
\pm 0.3\end{array}$ & $\begin{array}{r}93.4 \\
\pm 0.2\end{array}$ & $\begin{array}{c}95.1 \\
\pm 2.4\end{array}$ \\
\hline & 20 & $\begin{array}{r}95.8 \\
\pm 1.8 \\
\end{array}$ & $\begin{array}{c}97.3 \\
\pm 0.4 \\
\end{array}$ & $\begin{array}{r}97.2 \\
\pm 0.7 \\
\end{array}$ & $\begin{array}{r}97.8 \\
\pm 0.7 \\
\end{array}$ & $\begin{array}{c}97.4 \\
\pm 0.9\end{array}$ & $\begin{array}{r}95.6 \\
\pm 2.1 \\
\end{array}$ & $\begin{array}{r}96.5 \\
\pm 1.7 \\
\end{array}$ \\
\hline & 30 & $\begin{array}{r}96.6 \\
\pm 0.6 \\
\end{array}$ & $\begin{array}{r}96.2 \\
\pm 0.7 \\
\end{array}$ & $\begin{array}{r}97.8 \\
\pm 0.5\end{array}$ & $\begin{array}{r}96.9 \\
\pm 1.2 \\
\end{array}$ & $\begin{array}{r}97.9 \\
\pm 0.1 \\
\end{array}$ & $\begin{array}{r}97.7 \\
\pm 0.5 \\
\end{array}$ & $\begin{array}{r}96.0 \\
\pm 0.4 \\
\end{array}$ \\
\hline & 40 & $\begin{array}{r}96.6 \\
\pm 0.6 \\
\end{array}$ & $\begin{array}{r}96.8 \\
\pm 1.4 \\
\end{array}$ & $\begin{array}{r}98.7 \\
\pm 0.9\end{array}$ & $\begin{array}{r}97.9 \\
\pm 0.6\end{array}$ & $\begin{array}{r}98.7 \\
\pm 0.2 \\
\end{array}$ & $\begin{array}{r}98.3 \\
\pm 0.3 \\
\end{array}$ & $\begin{array}{r}97.4 \\
\pm 1.1 \\
\end{array}$ \\
\hline & 50 & $\begin{array}{r}96.6 \\
\pm 0.6 \\
\end{array}$ & $\begin{array}{r}97.9 \\
\pm 0.8 \\
\end{array}$ & $\begin{array}{r}98.7 \\
\pm 0.2 \\
\end{array}$ & $\begin{array}{r}97.6 \\
\pm 0.2 \\
\end{array}$ & $\begin{array}{r}98.5 \\
\pm 0.8 \\
\end{array}$ & $\begin{array}{r}98.5 \\
\pm 0.1 \\
\end{array}$ & $\begin{array}{c}97.9 \\
\pm 0.4 \\
\end{array}$ \\
\hline & 60 & $\begin{array}{r}98.2 \\
\pm 0.4 \\
\end{array}$ & $\begin{array}{c}99.1 \\
\pm 0.4 \\
\end{array}$ & $\begin{array}{r}99.0 \\
\pm 0.3 \\
\end{array}$ & $\begin{array}{r}98.5 \\
\pm 0.2 \\
\end{array}$ & $\begin{array}{r}98.6 \\
\pm 0.5 \\
\end{array}$ & $\begin{array}{r}98.7 \\
\pm 0.3 \\
\end{array}$ & $\begin{array}{r}98.5 \\
\pm 0.4 \\
\end{array}$ \\
\hline & 70 & $\begin{array}{r}98.4 \\
\pm 0.3 \\
\end{array}$ & $\begin{array}{r}98.8 \\
\pm 0.3 \\
\end{array}$ & $\begin{array}{r}99.2 \\
\pm 0.3 \\
\end{array}$ & $\begin{array}{r}98.7 \\
\pm 0.3 \\
\end{array}$ & $\begin{array}{r}99.2 \\
\pm 0.1 \\
\end{array}$ & $\begin{array}{r}99.2 \\
\pm 0.2 \\
\end{array}$ & $\begin{array}{r}98.7 \\
\pm 0.3 \\
\end{array}$ \\
\hline & 80 & $\begin{array}{r}99.0 \\
\pm 0.5 \\
\end{array}$ & $\begin{array}{c}99.1 \\
\pm 0.6 \\
\end{array}$ & $\begin{array}{r}99.0 \\
\pm 0.3 \\
\end{array}$ & $\begin{array}{r}99.0 \\
\pm 0.1 \\
\end{array}$ & $\begin{array}{c}99.1 \\
\pm 0.4\end{array}$ & $\begin{array}{r}99.3 \\
\pm 0.1 \\
\end{array}$ & $\begin{array}{r}98.7 \\
\pm 0.4 \\
\end{array}$ \\
\hline & 90 & $\begin{array}{r}99.2 \\
\pm 0.6 \\
\end{array}$ & $\begin{array}{r}98.9 \\
\pm 0.2\end{array}$ & $\begin{array}{r}99.3 \\
\pm 0.2 \\
\end{array}$ & $\begin{array}{r}99.2 \\
\pm 0.3\end{array}$ & $\begin{array}{c}99.4 \\
\pm 0.1\end{array}$ & $\begin{array}{r}99.4 \\
\pm 0.1 \\
\end{array}$ & $\begin{array}{r}99.3 \\
\pm 0.2 \\
\end{array}$ \\
\hline
\end{tabular}

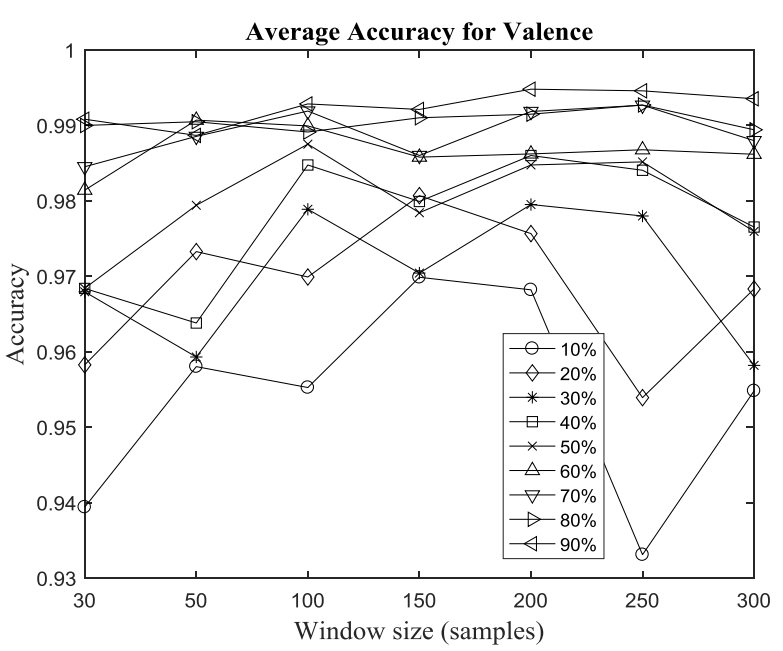

Figure 2. Average accuracy for Valence

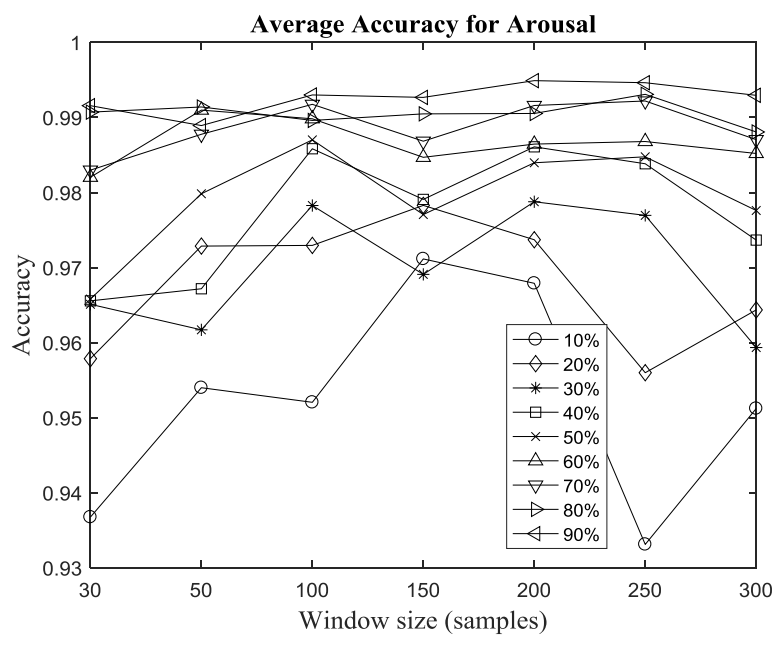

Figure 3. Average accuracy for Arousal

We also analyzed the performance based on the eight discrete emotional labels of the subjects from the database plus neutral, i.e. anger, disgust, fear, joy, sadness, surprise, amusement, and anxiety, see Table $\mathrm{V}$ for the average accuracies. The results also revealed that the proposed feature extraction method is also independent to discrete emotion of the subjects with the best result after 1000 repetitions occurred at 200 sample for window size with $90 \%$ overlap. The average accuracy for the whole experiment in discrete emotion is $97.9 \pm 1.4$. Fig. 4 also presents the same phenomenon as in valence and arousal by giving the best performance at $90 \%$ while $10 \%$ is the worst within the same window size parameters.

Experiments with reduced dimensionality to $15 \mathrm{D}$ showed similar results as without dimensionality reduction, with $92.8 \%$ as the lowest accuracy and $99.9 \%$ as the highest one when the emotional states of the subject were ignored. Once the emotions were considered, the lowest accuracy was $93.5 \%$ while the highest was $99.5 \%$. They had similar values as in the experiments without dimensionality reduction.

It can be considered as a weakness of our method that we did not specifically select the features on the basis of which information is relevant to biometry. It is left for future 
TABLE V. AVERAge PERFormances WHEN DISCRETE EMOTIONAL LABLES WERE CONSIDERED

\begin{tabular}{|c|c|c|c|c|c|c|c|c|}
\hline & \multicolumn{7}{|c|}{ Window size (samples) } \\
\hline & & 30 & 50 & 100 & 150 & 200 & 250 & 300 \\
\hline \multirow{9}{*}{$\frac{\int_{0}^{o}}{\frac{\sigma}{0}}$} & 10 & $\begin{array}{r}93.4 \\
\pm 2.9\end{array}$ & $\begin{array}{r}95.5 \\
\pm 2.8\end{array}$ & $\begin{array}{r}95.4 \\
\pm 3.0\end{array}$ & $\begin{array}{c}97.4 \\
\pm 1.4\end{array}$ & $\begin{array}{r}97.0 \\
\pm 1.3\end{array}$ & $\begin{array}{r}93.6 \\
\pm 3.3\end{array}$ & $\begin{array}{r}94.5 \\
\pm 2.7\end{array}$ \\
\hline & 20 & $\begin{array}{r}95.7 \\
\pm 2.6 \\
\end{array}$ & $\begin{array}{r}97.2 \\
\pm 1.5 \\
\end{array}$ & $\begin{array}{r}97.6 \\
\pm 1.6 \\
\end{array}$ & $\begin{array}{r}97.9 \\
\pm 1.8 \\
\end{array}$ & $\begin{array}{r}97.6 \\
\pm 1.3 \\
\end{array}$ & $\begin{array}{r}96.2 \\
\pm 3.3 \\
\end{array}$ & $\begin{array}{r}96.4 \\
\pm 3.1 \\
\end{array}$ \\
\hline & 30 & $\begin{array}{r}96.6 \\
\pm 2.0\end{array}$ & $\begin{array}{r}96.5 \\
\pm 1.7\end{array}$ & $\begin{array}{r}98.0 \\
\pm 1.0\end{array}$ & $\begin{array}{r}96.8 \\
\pm 2.5\end{array}$ & $\begin{array}{r}98.0 \\
\pm 1.1\end{array}$ & $\begin{array}{r}97.8 \\
\pm 1.8\end{array}$ & $\begin{array}{r}96.2 \\
\pm 2.2\end{array}$ \\
\hline & 40 & $\begin{array}{r}96.7 \\
\pm 2.0\end{array}$ & $\begin{array}{r}96.8 \\
\pm 1.9\end{array}$ & $\begin{array}{r}98.6 \\
\pm 0.8\end{array}$ & $\begin{array}{r}98.0 \\
\pm 1.4\end{array}$ & $\begin{array}{r}98.5 \\
\pm 0.8\end{array}$ & $\begin{array}{r}98.3 \\
\pm 0.5\end{array}$ & $\begin{array}{r}97.5 \\
\pm 1.6\end{array}$ \\
\hline & 50 & $\begin{array}{r}96.7 \\
\pm 1.7\end{array}$ & $\begin{array}{c}97.8 \\
\pm 1.1\end{array}$ & $\begin{array}{r}98.7 \\
\pm 0.5\end{array}$ & $\begin{array}{r}97.8 \\
\pm 1.5\end{array}$ & $\begin{array}{r}98.3 \\
\pm 1.6\end{array}$ & $\begin{array}{r}98.4 \\
\pm 0.7\end{array}$ & $\begin{array}{c}97.8 \\
\pm 1.0\end{array}$ \\
\hline & 60 & $\begin{array}{r}98.2 \\
\pm 0.9\end{array}$ & $\begin{array}{r}99.0 \\
\pm 0.9\end{array}$ & $\begin{array}{c}99.0 \\
\pm 0.6\end{array}$ & $\begin{array}{r}98.5 \\
\pm 1.2\end{array}$ & $\begin{array}{r}98.7 \\
\pm 0.8\end{array}$ & $\begin{array}{r}98.6 \\
\pm 0.4\end{array}$ & $\begin{array}{c}98.7 \\
\pm 1.1\end{array}$ \\
\hline & 70 & $\begin{array}{r}98.3 \\
\pm 1.2\end{array}$ & $\begin{array}{c}98.8 \\
\pm 0.8\end{array}$ & $\begin{array}{r}99.2 \\
\pm 0.5\end{array}$ & $\begin{array}{l}98.7 \\
\pm 0.5\end{array}$ & $\begin{array}{c}99.2 \\
\pm 0.6\end{array}$ & $\begin{array}{r}99.2 \\
\pm 0.5\end{array}$ & $\begin{array}{r}98.9 \\
\pm 1.1\end{array}$ \\
\hline & 80 & $\begin{array}{c}99.1 \\
\pm 0.6\end{array}$ & $\begin{array}{r}99.1 \\
\pm 0.7\end{array}$ & $\begin{array}{r}99.0 \\
\pm 0.5\end{array}$ & $\begin{array}{c}99.1 \\
\pm 0.6\end{array}$ & $\begin{array}{r}99.1 \\
\pm 0.9\end{array}$ & $\begin{array}{r}99.3 \\
\pm 0.5\end{array}$ & $\begin{array}{r}98.9 \\
\pm 1.1\end{array}$ \\
\hline & 90 & $\begin{array}{r}99.1 \\
\pm 1.0\end{array}$ & $\begin{array}{r}98.9 \\
\pm 0.5\end{array}$ & $\begin{array}{r}99.3 \\
\pm 0.5 \\
\end{array}$ & $\begin{array}{r}99.3 \\
\pm 0.5\end{array}$ & $\begin{array}{r}99.5 \\
\pm 0.3\end{array}$ & $\begin{array}{r}99.4 \\
\pm 0.5 \\
\end{array}$ & $\begin{array}{r}99.4 \\
\pm 0.6 \\
\end{array}$ \\
\hline
\end{tabular}

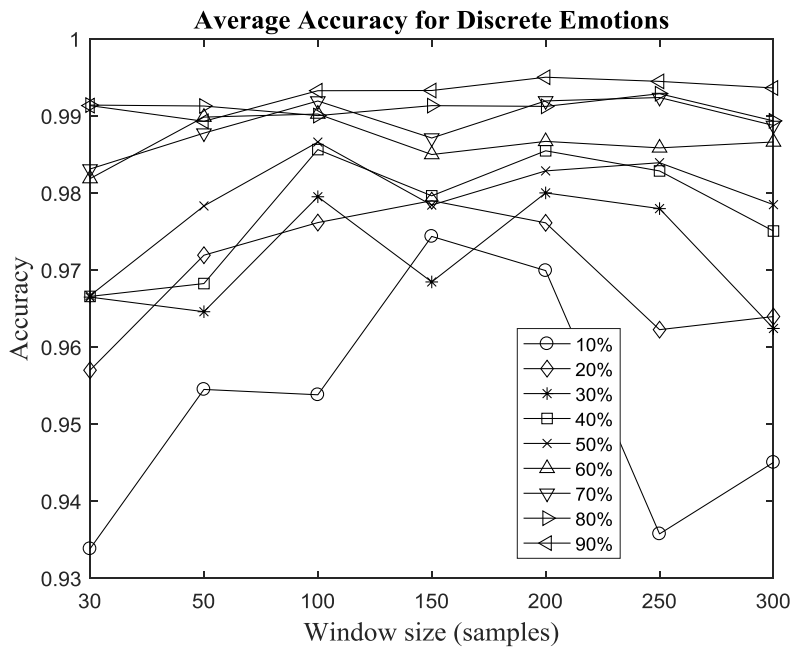

Figure 4. Average accuracy for discrete emotions

research, how the present features could be further developed to this direction.

\section{CONCLUSIONS}

The feature extraction method based on statistical distribution of dominant frequencies after applying the BEMD analysis to ECG signal was proposed. The experiments showed that the proposed method offers high accuracy with high consistency for ECG biometric identification. The experiments suggested $90 \%$ overlap parameter for very fine spectrogram analysis while nothing can be generalized for window size parameter.

The proposed method is also independent to the emotional states of the subject for both dimensional and discrete emotions. Having small variance among the results, it also indicates high consistency among the emotional states of the subjects. This characteristic is important in biometric identification as no one can have the same emotional state all the time. This finding suggests that other existing feature extraction methods need this kind of evaluation.

Although this study presents promising result, the generalizability of these results is subject to certain limitations related to the variation in ECG signal. For example, it is important to evaluate the proposed method under normal ECG vs. ECG with arrhythmias, ECG from various ages, and ECG from other affective recognition. Experiments with other non-linear classifiers are left for future work.

\section{REFERENCES}

[1] M. Hejazi, S. A. R. Al-Haddad, Y. P. Singh, S. J. Hashim and A. F. A. Aziz, "ECG biometric authentication based on non-fiducial approach using kernel methods," Digital Signal Processing, vol. 52, pp. 72-86, 2016.

[2] F. Sufi, I. Khalil and J. Hu, "ECG-based Authentication," in Handbook of Information and Communication Security, New York, Springer, 2010, pp. 309-331.

[3] F. Agrafioti, "ECG in Biometric Recognition: Time Dependency and Application Challenges [PhD Thesis]," Graduate Department of Electrical and Computer Engineering University of Toronto, Toronto, Canada, 2011.

[4] A. Fratini, M. Sansone, P. Bifulco and M. Cesarelli, "Individual identification via electrocardiogram analysis," BioMedical Engineering Online, vol. 14, no. 78, pp. 1-23, 2015.

[5] T. S. Lugovaya, "Biometric human identification based on electrocardiogram [Master's Thesis]," Faculty of Computing Technologies and Informatics, Electrotechnical University "LETI", Saint-Petersburg, Russian Federation, 2005.

[6] A. Page, A. Kulkarni and T. Mohsenin, "Utilizing deep neural nets for an embedded ECG-based biometric authentication system," in IEEE Biomedical Circuits and Systems Conference (BioCAS) 2015, Atlanta, GA, USA, 2015.

[7] M. Soleymani, J. Lichtenauer, T. Pun and M. Pantic, "A Multimodal Database for Affect Recognition and Implicit Tagging," IEEE Transactions on Affective Computing, vol. 3, no. 1, pp. 1-14, 2012.

[8] G. Rilling, P. Flandrin, P. Gonçalves and J. M. Lilly, "Bivariate Empirical Mode Decomposition," IEEE Signal Processing Letters, vol. 14, no. 12, pp. 936-939, 2007.

[9] P. E. McSharry, G. D. Clifford, L. Tarassenko and L. A. Smith, "A Dynamical Model of Generating Synthetic Electrocardiogram Signals," IEEE Transactions on Biomedical Engineering, vol. 50, no. 3, pp. 289-294, 2003.

[10] J. Pan and W. J. Tompkins, "A Real-Time QRS Detection Algortihm," IEEE Transactions on Biomedical Engineering, Vols. BME-32, no. 3, pp. 230-236, 1985.

[11] H. Ferdinando, T. Seppänen and E. Alasaarela, "Comparing Features from ECG Pattern and HRV Analysis for Emotion Recognition System," in The annual IEEE International Conference on Computational Intelligence in Bioinformatics and Computational Biology (CIBCB 2016), Chiang Mai, Thailand, 2016.

[12] F. Agrafioti, D. Hatzinakos and A. K. Anderson, "ECG Pattern Analysis for Emotion Detection," IEEE Transactions on Affective Computing, vol. 3, no. 1, pp. 102-115, 2012.

[13] F. Agrafioti and D. Hatzinakos, "An enhanced EMD algorithm for ECG signal processing," in 17th International Conference on Digital Signal Processing (DSP), Corfu, 2011.

[14] H. Ferdinando, T. Seppänen and E. Alasaarela, "Enhancing Emotion Recognition from ECG Signals using Supervised Dimensionality Reduction," in Proceeding of the 6th International Conference on Pattern Recognition Application and Methods (ICPRAM), Porto, Portugal, 2017. 